\title{
A comment on Bonnor-Steadman closed timelike curves
}

\author{
Valéria M. Rosa • Patricio S. Letelier
}

Received: 1 April 2008 / Accepted: 20 August 2008 / Published online: 7 September 2008 (C) Springer Science+Business Media, LLC 2008

\begin{abstract}
The existence and stability closed timelike curves in a Bonnor-Ward spacetime without torsion line singularities is shown by exhibiting particular examples.
\end{abstract}

Keywords Closed timelike curves $\cdot$ Stability $\cdot$ Torsion lines $\cdot$ Singularities

In a seminal paper Bonnor and Steadman [1] study the existence of closed timelike curves (CTC) in the Bonnor-Ward metric [2] whose source are two Perjeons (see below) with masses $m_{1}$ and $m_{2}$, and magnetic moments $\mu_{1}$ and $\mu_{2}$ placed on the $z$-axis at $z= \pm a,(a>0)$. The source is bounded and the spacetime is asymptotically flat. In principle, this spacetime is physically realizable [1]. Furthermore, in this spacetime we have regions with stable closed timelike geodesics (CTGs) [3].

In the spacetime for the already studied cases of CTCs and CTGs, besides the singularities associated the punctual masses that in the particular examples studied are equal, we ought to have a torsion line singularity (TLS) [4,5] joining the two sources. If one eliminates the TLS we also kill the CTCs and CTGs. One can argue that physically this is not objetionable because one can thought this singularities as the mathematical representation of a mechanical device to keep the two particles fixed. But, since on a TLS the geometry of the spacetime is no longer Riemannian these singularities are rather pathological.

\footnotetext{
V. M. Rosa

Departamento de Matemática, Universidade Federal de Viçosa, 36570-000 Viçosa, MG, Brazil

e-mail: vmrosa@ufv.br

P. S. Letelier $(\varangle)$

Departamento de Matemática Aplicada-IMECC,

Universidade Estadual de Campinas,

13081-970 Campinas, SP, Brazil

e-mail: letelier@ime.unicamp.br
} 
In this comment we show that we can have CTCs in a spacetime without TLS when the point particles (the source of the spacetime) are not equal. This makes the appearance of CTCs in the Bonnor-Ward spacetime more worrisome.

The Bonnor-Ward spacetime is a particular case of the Perjés-Israel-Wilson (PIW) metric $[6,7]$,

$$
d s^{2}=-f^{-1} h_{m n} d x^{m} d x^{n}+f\left(\omega_{m} d x^{m}+d t\right)^{2},
$$

where the three dimensional positive definite tensor $h_{m n}$ has zero Ricci tensor and it is usually taken as the three dimensional Euclidean metric in cylindrical coordinates. The punctual sources of the PIW spacetime are named Perjeons [2]. A Perjeon has mass $m$, electric charge $e$, classical angular momentum $\vec{h}$ and magnetic moment $\vec{\mu}$ related, in relativistic units, by the equations: $m=\epsilon e$ and $\vec{h}=\epsilon \vec{\mu}$, where $\epsilon= \pm 1$.

We shall consider the particular case of the Bonnor-Ward solution described in [1] with a small modification. In the definition of function $\Omega\left(\omega_{a}=-\rho^{2} \Omega \delta_{a}^{\varphi}\right)$ we impose that $\alpha \equiv m_{1} \mu_{2}+m_{2} \mu_{1}=0$. In this case, the Bonnor-Ward solution does not present TLS. Note that in the cases of CTCs and CTG studied in [2] $\alpha \neq 0$.

A closed curve $\gamma$ in this spacetime is given in its parametric form by, $t=t_{0}, \rho=\rho_{0}$, $\varphi \in[0,2 \pi], z=z_{0}$, where $t_{0}, \rho_{0}$ and $z_{0}$ are constants. This closed curve is timelike when $g_{\varphi \varphi}>0$, i.e., when $\Omega^{2} f^{2} \rho^{2}-1>0$. The four-acceleration $a^{\mu}$ of $\gamma$ has two nonzero components, $a^{\rho}$ and $a^{z}$.

In [1] it is shown that when $m_{1}=m_{2}, \mu_{1}=-\mu_{2}$ and $z_{0}=0$, we have $\alpha=0$, but in this case we have $g_{\varphi \varphi}\left(\rho_{0}, z_{0}\right)=-\rho_{0}^{2} / f$. Therefore the closed curve $\gamma$ is not timelike. If we weaken these restrictions, for instance, taking $m_{2}=n m_{1}, \mu_{2}=-n \mu_{1}$ with $n \neq 1$, we have $\alpha=0$ and it is possible to find CTCs.

The linear stability of a curve $\gamma$ is studied by adding a perturbation $\xi$ to $\left(t_{0}, \rho_{0}, \varphi, z_{0}\right)$ and analyzing the behavior of this perturbation through the modified equation of geodesic deviation presented in [3]. For the CTC $\gamma$ we have that this equation reduces to a system of second order ordinary differential equation with constant coefficients. This system has bound solutions (periodic modes) when their eigenvalues, $\lambda$, are pure imaginary numbers, $\left(\lambda_{1}= \pm \imath \kappa_{1}, \lambda_{2}= \pm \imath \kappa_{2}\right)$. The explicit forms of $\kappa_{1}$ and $\kappa_{2}$, depending on the distance $a$, the parameters $m_{1}, \mu_{1}, n$, and the position $\left(\rho_{0}, z_{0}\right)$, are rather cumbersome and will be presented elsewhere.

We find that when the parameters are: $a=5, \rho_{0}=12, m_{1}=0.1, n=0.01$, $\mu_{1}=1,800$, and $z_{0}=-0.1$, the closed curve $\gamma$ is timelike $\left(g_{\varphi \varphi}=254.03612\right)$ and stable $\left(\kappa_{1}=0.1712868682\right.$ and $\left.\kappa_{2}=0.9902631725\right)$. For this example the values of acceleration are: $a^{\rho}=0.1623818860$, and $a^{z}=0.1737327461$. We get another example of a stable CTC when $a=6, \rho_{0}=8, m_{1}=0.1, n=0.01, \mu_{1}=209.32$, and $z_{0}=0$. For these values of the parameters, we have $g_{\varphi \varphi}=102.0184576, \kappa_{1}=$ $0.09159526892, \kappa_{2}=0.4104757936, a^{\rho}=0.1718928982$ and $a^{z}=0.1719035561$. A third example of stable CTC is obtained when $a=3, \rho_{0}=9.39, m_{1}=0.1$, $n=0.001, \mu_{1}=1,900$, and $z_{0}=0$. In this case, we get $g_{\varphi \varphi}=160.9211303, \kappa_{1}=$ $0.5441422796, \kappa_{2}=1.176146266 a^{\rho}=0.2139746920$ and $a^{z}=0.3157680388$.

To find a CTC $\gamma$ in this spacetime is not difficult, we only need $g_{\varphi \varphi}>0$. But, to get a stable one $\left(\lambda_{1}^{2}<0\right.$, and $\left.\lambda_{2}^{2}<0\right)$ is not so simple. We need to have one positive 
function and two negative ones, our numerical experiments indicates that these seldom occurs.

The examples of CTC with no TLS presented in this comment indicates a possible existence of closed timelike geodesics in the Bonnor-Ward solution. To find a explicit example of these curves is not an easy task, we need: $g_{\varphi \varphi}>0$, and $a^{\rho}=a^{z}=0$. If we add the condition of stability: $\lambda_{1}^{2}<0$, and $\lambda_{2}^{2}<0$ (pure imaginary eivenvalues), we believe that will be almost impossible to satisfy all these conditions. In summary, we conjecture that stable closed timelike geodesics in the Bonnor-Ward solution without TLS do no exist.

Finally, we want to report that we looked for closed timelike geodesics in a perturbed Bonnor-Ward solution. We found that our multipolar perturbations introduces TLS.

Acknowledgments Rosa VM thanks the hospitality of DMA-IMECC-UNICAMP. Letelier PS thanks the partial financial support of FAPESP and CNPq.

\section{References}

1. Bonnor, W.B., Steadman, B.R.: Gen. Relativ. Gravit. 37, 1833 (2005)

2. Bonnor, W.B., Ward, J.P.: Commun. Math. Phys. 28, 323 (1972)

3. Rosa, V.M., Letelier, P.S.: Phys. Lett. A 370, 99 (2007)

4. Letelier, P.S., Oliveira, S.R.: Phys. Lett. A 238, 101 (1998)

5. Letelier, P.S.: Class. Quantum. Gravit. 12, 471 (1995)

6. Perjés, Z.: Phys. Rev. Lett. 27, 1668 (1971)

7. Israel, W., Wilson, G.A.: J. Math. Phys. 13, 323 (1972) 